\title{
Vorprüfung, Sachentscheidungsvoraussetzungen oder Zulässigkeit? Gutachtenaufbau in der öffentlich-rechtlichen Klausur
}

\author{
Von Wiss. Mit. Martin Heidebach, München*
}

Der Beitrag beschäftigt sich mit einer Grundfrage des Gutachtenaufbaus in der öffentlich-rechtlichen Klausur, die immer wieder zu Verunsicherung bei den Studierenden führt. Zunächst wird die Ursache für die verschiedenen Aufbauvarianten dargestellt. Anschließend wird geprüft, welche der Aufbaumöglichkeiten die überzeugendste ist. Bei dieser tiefer gehenden Betrachtung wird sich herausstellen, dass der klassische Aufbau in Zulässigkeit und Begründetheit vorzugswürdig bleibt.

\section{Einleitung}

Eigentlich gehört es zu den Grundkonstanten des öffentlichrechtlichen Gutachtens, dass zumindest der Prüfungsaufbau der Zulässigkeit einem klar strukturierten Schema folgt. Dies vermittelt den Bearbeiterinnen und Bearbeitern eine gewisse Sicherheit im Umgang mit öffentlich-rechtlichen Klausuren ${ }^{1}$, denn zumindest deren erster Teil lässt sich so zumeist in den Griffbekommen ${ }^{2}$. Bei genauerer Betrachtung ergeben sich aber auch in der Zulässigkeitsprüfung einige grundsätzliche Fragen, deren Beantwortung die Studierenden vor eine Herausforderung stellt ${ }^{3}$. Dabei geht es weniger darum, dass es sich um besonders komplizierte Probleme handelt. Vielmehr liegt die Schwierigkeit darin, dass von den Studierenden erwartet wird, dass sie stets die richtigen Antworten wählen, zugleich diese aber nicht erläutern sollen. Es gilt der Grundsatz, dass sich der Prüfungsaufbau von selbst erklären soll und daher keiner Begründung bedarf ${ }^{4}$. Zugleich befinden sich die Klausurbearbeitenden aber in dem Dilemma, dass es ihnen der Korrektor sehr übel nehmen kann, wenn sie einen von diesem als falsch angesehenen Aufbau wählen. Besonders zeigt sich dies anhand der Problematik der Einordnung der Prüfungspunkte Verwaltungsrechtswegeröffnung nach $\$ 40$ I 1 VwGO und sachlicher und örtlicher Zuständigkeit des Gerichts in den Prüfungsaufbau, um die es im Folgenden gehen soll. Traditionell gliedert sich die Prüfung eines Rechtsbehelfs in Zulässigkeit und Begründetheit. Immer öfter liest man in öffentlich-rechtlichen Klausuren die Unterteilung in Sachentscheidungsvoraussetzungen und Begründetheit. Schließlich wird von manchen der Zulässigkeit- und Begründetheitsprüfung eine Vorprüfung vorangestellt ${ }^{5}$.

Ziel des Aufsatzes ist es, den Studierenden die Sicherheit im Hinblick auf diese Aufbaufrage zurückzugeben, die durch die unterschiedlichen Sichtweisen scheinbar verloren gegangen ist ${ }^{6}$.

Zunächst soll nochmals die Ursache für die Problematik dar- gestellt werden. Im Anschluss setzt sich der Aufsatz mit den verschiedenen Aufbauvarianten auseinander und prüft, welche die überzeugendste Lösung bietet.

\section{Grund für die Verunsicherung}

Wie bereits erwähnt, wird bei der Prüfung eines Rechtsbehelfs nicht nur im öffentlichen Recht, sondern in allen Rechtsgebieten - klassischerweise zwischen Zulässigkeit und Begründetheit unterschieden. Diese Unterscheidung wird für so selbstverständlich gehalten, dass sie in den jeweiligen Prozessordnungen nicht ausdrücklich geregelt ist ${ }^{7}$. Nach allgemeinem Verständnis prüft das Gericht im Rahmen der Zulässigkeit, ob es überhaupt eine Ent-

* Der Verfasser ist wissenschaftlicher Mitarbeiter am Lehrstuhl von Prof. Dr. Dres. h.c. Hans-Jürgen Papier an der LMU München.

1 Mit »öffentlich-rechtlicher « Klausur sind im Rahmen des vorliegenden Aufsatzes Gutachten über verwaltungsgerichtliche Streitigkeiten gemeint, die die weit überwiegende Mehrzahl der öffentlich-rechtlichen Klausuren darstellen. Bei verfassungsrechtlichen Klausuren finden sich die hier erläuterten Probleme nicht, da $\$ 17$ a II GVG (vgl sogleich unter II.) für die Verfassungsgerichtsbarkeit nicht anwendbar ist (Sodan/Ziekow/ZıEKOw Verwaltungsgerichtsordnung, 2. Aufl 2006, $\$ 17$ GVG Rdn.4). Bei diesen Klausuren ist es unstreitig, dass der Aufbau in Zulässigkeit und Begründetheit zu erfolgen hat.

2 Leider verleitet dies die Studierenden oftmals dazu, den Schwerpunkt der Arbeit auf die Zulässigkeitsprüfung zu legen, weil sie sich dort an einem bekannten Gerüst orientieren können.

3 Ein weiteres Beispiel für ein derartiges Problem findet sich bei MeInHard Schröder Bundes- oder Landes-VwVfG bei Definitionslücken in der VwGO?, JA 2009 (im Erscheinen).

4 Stern/Blanke Verwaltungsprozessrecht in der Klausur, 9. Aufl 2008, Rdn. 130.

5 Überraschenderweise wird nicht einmal bei den Lösungsskizzen zu den Klausuren des Ersten Juristischen Staatsexamens in Bayern ein einheitlicher Aufbau vertreten, wie eine Analyse der in den BayVBl veröffentlichten Klausuren der Examina aus den Jahren 2000 bis 2004 ergeben hat. Allerdings dominiert immer noch der klassische Aufbau in Zulässigkeit und Begründetheit.

6 Die Verunsicherung der Studierenden kann der Verfasser aus eigener Erfahrung als Dozent des Examinatoriums öffentliches Recht im Rahmen des Münchner Examentrainings an der LMU bestätigen.

7 Sie ergibt sich auch implizit aus den vereinzelten Regelungen in der VwGO, etwa $\$ \$ 42$ II, 75 S. 1, 109, vgl Schoch/Schmidt-Aßmann/Pietzner/ EHLERS Verwaltungsgerichtsordnung, Vorb $\$ 40 \mathrm{Rdn} .2$. 
scheidung in der Sache treffen $\mathrm{kann}^{8}$. Erst im Rahmen der Begründetheit entscheidet das Gericht inhaltlich über den Rechtsstreit ${ }^{9}$. Dementsprechend kann ein Rechtsbehelf sowohl wegen Unzulässigkeit als auch aufgrund von Unbegründetheit scheitern.

Die traditionellen Ausgangsfragen eines Verwaltungsgerichts bei der Zulässigkeitsprüfung eines Rechtsbehelfs waren, ob für den zu behandelnden Fall der Rechtsweg zu dem entsprechenden Gericht eröffnet und ob das Gericht für die Frage sachlich und örtlich zuständig ist. Für öffentlich-rechtliche Streitigkeiten richtet sich die Rechtswegeröffnung dabei nach $\$ 40$ I 1 VwGO und die Frage der sachlichen und örtlichen Zuständigkeit nach $\$ 45$ VwGO bzw. $\$ 52$ VwGO i. V. m. dem jeweiligen Landesgerichtsorganisationsgesetz. Kam das Gericht zu dem Ergebnis, dass der Rechtsweg z. B. im Hinblick auf eine Klage nicht eröffnet oder seine Zuständigkeit für diese nicht gegeben war, so war die Klage unzulässig und wurde vom Gericht abgewiesen ${ }^{10}$.

Mit dem Vierten Gesetz zur Änderung der VwGO vom 17. 12.1990 beseitigte der Gesetzgeber diese Möglichkeit der Klageabweisung im Interesse der Vereinfachung des Verfahrens und der Kostenersparnis ${ }^{11}$. Die (erstinstanzlichen) ${ }^{12}$ Gerichte müssen zwar weiterhin die Rechtswegeröffnung prüfen ${ }^{13}$. Kommen sie aber zu dem Ergebnis, dass der Rechtsweg zu ihrem Gericht nicht gegeben ist, so sind sie durch die Neufassung des $\$ 17$ a II 1 GVG verpflichtet, den Rechtsstreit an das zuständige Gericht des richtigen Rechtswegs zu verweisen, für das die Verweisung gem. $\$ 17$ a II 3 GVG in dieser Hinsicht bindend ist ${ }^{14}$. Eine Abweisung der Klage aufgrund von Unzulässigkeit wegen fehlender Rechtswegeröffnung nach $\$ 40 \mathrm{I} 1 \mathrm{VwGO}$ ist daher nicht mehr möglich. Gleiches gilt, wenn das Gericht feststellt, dass es sachlich oder örtlich unzuständig ist. Gem. $\$ 83 \mathrm{VwGO}$ sind die Vorschriften der $\$ \$ 17$ bis $17 \mathrm{~b}$ GVG auf die sachliche und örtliche Zuständigkeit entsprechend anzuwenden ${ }^{15}$. Auch in diesen Fällen kann durch das Gericht also allenfalls ein Verweisungsbeschluss ergehen, eine Klageabweisung ist nicht vorgesehen.

Zum Teil wird bestritten, dass die $\$ 17$ bis 17 b GVG auch auf die Rechtsbehelfe des einstweiligen Rechtsschutzes ( $\$ 80 \mathrm{~V}, \$ 123$ VwGO) anzuwenden sind ${ }^{16}$. Nach überwiegender Ansicht wird eine Differenzierung zwischen Eilrechtsschutz und Klageverfahren im Hinblick auf die Anwendung der Vorschriften jedoch abgelehnt ${ }^{17}$, so dass auch insoweit eine Zurückweisung des Antrags als unzulässig in den genannten Fällen nicht mehr möglich ist.

\section{Aufbaumöglichkeiten}

Nachdem die Ursache dargestellt wurde, aufgrund derer der klassische Prüfungsaufbau in Zulässigkeit und Begründetheit in Frage gestellt wird, soll nun den verschiedenen Aufbauvarianten nachgegangen werden und diese sollen auf ihre Tauglichkeit hin überprüft werden.

\section{Vorprüfung}

Einige Autoren schlagen als Lösung des Aufbauproblems vor, die Prüfungspunkte "Rechtswegeröffnung " und "Zuständigkeit vor die Zulässigkeitsprüfung zu ziehen und dort unter der Überschrift »Vorprüfung « ${ }^{18}$ abzuhandeln ${ }^{19}$. Deshalb wird dies auch als dreistufiger Aufbau bezeichnet (Vorprüfung - Zulässigkeit Begründetheit). Ihr Ausgangspunkt ist, dass ein Rechtsbehelf wegen fehlender Rechtswegeröffnung oder Zuständigkeit nicht mehr als unzulässig abgewiesen werden könne. Sei dies der Fall, so könnten die beiden Fragen keine Zulässigkeitsvoraussetzung mehr darstellen und daher auch nicht mehr im Rahmen der Zulässigkeit geprüft werden.

Auf den ersten Blick scheint dieser Aufbau zu überzeugen und die Änderung des $\$ 17$ a GVG in angemessener Form aufzunehmen. a) Allerdings weißt dieses Konzept bereits praktische Schwierigkeiten auf.

Zum einen ist unklar, wie man den Obersatz bilden soll. Traditionell lautet dieser: »Die Klage ist erfolgreich, wenn sie zulässig und begründet ist«. Jedenfalls passt es nicht, die Vorprüfung in diesen Obersatz aufzunehmen, denn das Ergebnis der Vorprüfung hat nach dem oben Gesagten keine Auswirkungen auf den Erfolg der Klage ${ }^{20}$ : Stellt sich heraus, dass der Rechtsweg nicht eröffnet ist oder das Gericht nicht zuständig ist, so wird es den Rechtsstreit nach $\$ 17$ a II GVG an das zuständige Gericht verweisen. Wählt man diesen Aufbau, so sollte man wohl am besten folgendermaßen beginnen: »Der Kläger erhebt Klage vor dem Verwaltungsgericht, daher ist zunächst im Rahmen einer Vorprüfung zu prüfen, ob der Rechtsweg nach $\$ 40$ I 1 VwGO eröffnet und ob das Gericht sachlich und örtlich zuständig ist«. Nach der Vorprüfung sollte dann der oben zitierte traditionelle Obersatz folgen.

Zum anderen führt es zu Problemen, wenn man die Zuständigkeitsprüfung vor die Statthaftigkeitsprüfung zieht. Für die sachliche Zuständigkeit mag dies nicht so gravierend sein, da gem. $\$ 45 \mathrm{VwGO}$ grundsätzlich das Verwaltungsgericht entscheidet. Die örtliche Zuständigkeit knüpft sich nach $\$ 52 \mathrm{Nr}$. 2, 3 und 5 VwGO jedoch an die Klageart, so dass sich hier praktisch immer die Frage nach dieser stellt ${ }^{21}$. Natürlich geht es im $\mathrm{Zu}-$ sammenhang mit der Prüfung der örtlichen Zuständigkeit nicht darum, ob die gewählte Klageart statthaft ist. Unproblematisch ist es daher, wenn im Sachverhalt klar bezeichnet ist, welche Art von Klage der Kläger erhebt. Nur ist dies zumeist nicht der Fall; in den meisten Klausuren heißt es schlicht, dass die Erfolgsaus-

$8 \mathrm{Vgl}$ nur Kopp/Schenke VwGO, 15. Aufl 2007, Vorb $\$ 40$ Rdn. 2; Sodan/ Ziekow/Sodan Verwaltungsgerichtsordnung, 2. Aufl 2006, \$ 40 Rdn. 45.

9 Dies bedeutet nicht, dass gewisse Sachfragen bereits bei der Zulässigkeitsprüfung keine Rolle spielen, etwa im Hinblick auf den Rechtsweg und die Klagebefugnis. Die Entscheidung über die Sachfrage selbst bleibt aber der Begründetheitsprüfung überlassen.

10 Kissel NJW 1991, 947, 949. Auf Antrag des Klägers konnte das Gericht den Rechtsstreit an das (seiner Auffassung nach) zuständige Gericht verweisen.

11 BT-Drcks 11/7030, S. 37

$12 \mathrm{Vgl} \$ 17$ a V GVG.

13 Kopp/Schenke VwGO, 15. Aufl 2007, Vorb $\$ 40$ Rdn. 1.

14 Vgl Kissel NJW 1991, 947, 949. Das GVG gilt für den Verwaltungsprozess gem $\$ 173 \mathrm{~S} .1$ VwGO.

15 Kopp/Schenke VwGO, 15. Aufl 2007, \$83 Rdn. 7. Daneben ist $\$ 83$ VwGO auch bei instanzieller Unzuständigkeit anzuwenden, REDEKER/ von Oertzen Verwaltungsgerichtsordnung, 14. Aufl 2004, \$83 Rdn. 1.

$16 \mathrm{Kopp} /$ Schenke VwGO, 15. Aufl 2007, $\$ 41$ Rdn. 2 a. Folgt man dieser Meinung, so bleibt es schon von daher im Hinblick auf die Rechtsbehelfe des einstweiligen Rechtsschutzes beim traditionellen Aufbau in Zulässigkeit und Begründetheit.

17 Vgl nur BayVGH NVwZ-RR 2003, 74; Sodan/Ziekow/Puttler Verwaltungsgerichtsordnung, 2. Aufl 2006, $\$ 80$ Rdn. 113. Diese Ansicht überzeugt, da sich für eine Differenzierung auch keinerlei Anhaltspunkte in den Vorschriften der $\$ \$ 17$ bis 17 b GVG finden.

18 Bisweilen wird dieser Punkt auch als "Entscheidungskompetenz« bezeichnet, vgl zB Lösungsskizze Aufgabe 7 der Ersten Juristischen Staatsprüfung in Bayern, Termin 2004/1, BayVBl 2007, 188, 189.

19 Vgl Hufen Verwaltungsprozessrecht, 7. Aufl 2008, \$10 Rdn. 1.

20 Ein solcher Obersatz findet sich aber in der Lösungsskizze zu einer Examensklausur das Ersten Staatsexamens in Bayern (Aufgabe 6 aus dem Termin 2004/1, BayVBl 2007, 59). Die Verunsicherung im Hinblick auf den Obersatz zeigt sich in dieser Klausur auch darin, dass er lautet »Die Klage hat Aussicht auf Erfolg, wenn der Verwaltungsrechtsweg eröffnet ist und die Klage zulässig und begründet ist «. Die Frage der Zuständigkeit wird dagegen nicht angesprochen, dann aber im Rahmen der Vorprüfung neben der Rechtswegeröffnung geprüft. Zwei weitere Beispiele aus dem Ersten Bayerischen Staatsexamen verdeutlichen die Probleme mit dem Obersatz. In den beiden Klausuren wird der traditionelle Obersatz (Klage erfolgreich, wenn zulässig und begründet) über die Vorprüfung gesetzt (Aufgabe 7 aus dem Termin 2004/1, BayVBl 2007, 188 f; Aufgabe 8 aus dem Termin 2004/1, BayVBl 2007, 379). Belässt man es bei diesem Obersatz bleibt unklar, warum als erstes eine Vorprüfung erfolgt.

21 FisCHER JURA 2003, 748 
sichten der erhobenen Klage zu prüfen sind, weil das Ermitteln der richtigen Klageart im Rahmen der Statthaftigkeitsprüfung von den Kandidatinnen und Kandidaten gerade erwartet wird. Wählt man den dreistufigen Aufbau, so steht man folglich in den allermeisten Klausuren vor dem Dilemma der Vorgreiflichkeit der Statthaftigkeitsprüfung zumindest für die örtliche Zuständigkeit.

b) Auch inhaltlich kann der dreistufige Aufbau nicht überzeugen, wenn man die oben angeführte Prämisse einer genaueren Prüfung unterzieht. Nach dieser bedarf es einer Vorprüfung, da Rechtswegeröffnung und Zuständigkeit wegen der Neuregelung des $\$ 17$ a II GVG und der damit zusammenhängenden Verweisungspflicht keine Zulässigkeitsvoraussetzungen mehr seien. Dem liegt die These zugrunde, dass ein bestimmtes Merkmal nur dann zu den Zulässigkeitsvoraussetzungen gerechnet werden kann, wenn dessen Nichtvorliegen zwingend die Abweisung des Rechtsbehelfs nach sich zieht ${ }^{22}$.

Der Prämisse können verschiedene Argumente entgegengehalten werden:

aa) Zunächst lässt sich für den Prüfungspunkt Rechtswegeröffnung vorbringen, dass es in der - oben dargestellten - Totalität nicht ganz richtig ist, dass das Gericht den Rechtsstreit nach $\$ 17$ a II GVG stets verweisen muss, wenn die Voraussetzungen des $₫ 40$ I 1 VwGO nicht vorliegen. Dies gilt nämlich nur, soweit es sich nicht um eine öffentlich-rechtliche Streitigkeit handelt. Ist der Rechtsstreit dagegen öffentlich-rechtlicher Natur, aber verfassungsrechtlicher Art, so greift $\$ 17$ a II GVG nicht ein ${ }^{23}$. Hintergrund der Regelung der $\$ \$ 17$ bis 17 b GVG ist die Existenz von fünf verschiedenen Rechtswegen für einfachrechtliche Streitigkeiten; die Normen dienen dazu, diese Problematik $\mathrm{zu}$ entschärfen ${ }^{24}$. Folglich sind sie auf verfassungsrechtliche Streitigkeiten nicht anwendbar, da es insoweit nur - wenn überhaupt - den Rechtsweg zur Verfassungsgerichtsbarkeit gibt. Ist der verwaltungsgerichtliche Rechtsweg aus diesem Grund nicht eröffnet, so muss das Gericht den Rechtsbehelf (als unzulässig) abweisen $^{25}$. Streng genommen müsste man also - wenn man dem dreistufigen Aufbau folgt - die Prüfung des $\$ 40$ I 1 VwGO in zwei Komplexe aufspalten und die Frage nach dem verfassungsrechtlichen Charakters der Streitigkeit als Zulässigkeitsvoraussetzung prüfen.

Ähnlich verhält es sich bei der Normenkontrolle nach $\$ 47$ VwGO. Der $\$ 17$ a II VwGO ist für diesen Fall zwar anwendbar, allerdings findet sich kein Gericht, das für nicht-öffentlichrechtliche prinzipale Normenkontrollen zuständig wäre. Da eine Verweisung nicht möglich ist, kommt auch hier nur die Abweisung als unzulässig in Betracht, wenn die Vorgaben des $\$ 40$ I 1 VwGO nicht vorliegen ${ }^{26}$. Wird ein Antrag auf Normenkontrolle gestellt, so müssen also auch die Vertreter des dreistufigen Aufbaus den klassischen zweistufigen Aufbau verwenden.

bb) Eine weitere Inkonsequenz des dreistufigen Aufbaus zeigt sich darin, dass nicht auch die Statthaftigkeitsprüfung unter den Punkt Vorprüfung gefasst wird. Nach der dem dreistufigen Aufbau zugrunde liegenden Definition von Zulässigkeitsvoraussetzung dürfte auch diese bei den meisten Gutachten nicht in die Zulässigkeitsprüfung fallen. Bei der Prüfung der Statthaftigkeit der Klage geht es um die Frage, welche Art der Klage der klägerische Antrag beinhaltet. Die Klage kann dabei bloß dann wegen Unzulässigkeit abgewiesen werden, wenn der Kläger sich explizit auf die falsche Klageart stützt ${ }^{27}$. Wie bereits oben dargestellt wird in den meisten Klausurfällen dagegen nur das Begehren des Klägers umschrieben und die Bearbeiter müssen unter dem Punkt Statthaftigkeit prüfen, welche Klageart die richtige ist. In diesen Fällen des Gutachtens kann die Prüfung nicht zu dem Ergebnis kommen, dass die Klage wegen fehlender Statthaftigkeit als unzulässig abgewiesen wird. Aufgrund der Generalklausel des $\$ 40$ I 1 VwGO - die die Vorgaben des Art. 19 IV 1 GG widerspiegelt $^{28}$ - muss es für jedes klägerische Begehren, für das der Rechtsweg zur Verwaltungsgerichtsbarkeit eröffnet ist, auch eine statthafte Klageart geben ${ }^{29}$. Auch in der Praxis ist der Kläger nicht gehalten, in seinem Antrag die gewünschte Klageart genau $\mathrm{zu}$ bezeichnen. Es genügt gem. $\$ \$ 82$ I 2, $88 \mathrm{VwGO}$, wenn sich aus dem Klagebegehren und dem Klageantrag die richtige Klageart durch Auslegung ermitteln lässt ${ }^{30}$.

Würden die Vertreter des dreistufigen Aufbaus ihren eigenen Prämissen konsequent folgen, so dürften sie die Statthaftigkeitsprüfung nur unter die Zulässigkeit subsumieren, wenn der Kläger in seinem Antrag eindeutig eine bestimmte Klageart bezeichnet, ohne dass das Klagebegehren einer anderweitigen Auslegung zugänglich ist.

cc) Dieser Umstand weist darauf hin, dass die Grundthese zu hinterfragen ist, wonach zur Zulässigkeit nur solche Merkmale gehören, deren Nichtvorliegen die Rechtsbehelfsabweisung nach sich zieht. Diese stellt nämlich einen Zirkelschluss dar ${ }^{31}$ : Der Rechtsbehelf ist als unzulässig abzuweisen, wenn die Zulässigkeitsmerkmale nicht gegeben sind. Zulässigkeitsmerkmale definieren sich aber als die Prüfungspunkte, deren Fehlen zur Abweisung des Rechtsbehelfs als unzulässig führt. Einen Erkenntniswert hat diese Definition deshalb nicht. Vielmehr sollte man sich auf das eingangs erwähnte traditionelle Verständnis der Zulässigkeitsprüfung besinnen, nach dem das Gericht im Rahmen der Zulässigkeit prüft, ob es überhaupt zu einer Entscheidung in der Sache kommen kann ${ }^{32}$. Zulässigkeitsmerkmale sind folglich alle Fragen, die vom angerufenen Gericht zu prüfen sind, bevor es eine Prüfung in der Sache durchführen darf ${ }^{33}$. Nach dieser Definition gehören Rechtswegeröffnung und Zuständigkeit zur Zulässigkeit des Rechtsbehelfs, denn sie sind Voraussetzung dafür, dass das angerufene Gericht eine Sachentscheidung trifft $^{34}$. Die Neuregelung des $\$ 17$ a II GVG bzw. des $₫ 83$ VwGO hat deshalb den Charakter der Rechtswegeröffnung und der $\mathrm{Zu}$ ständigkeitsprüfung nicht verändert, sondern normiert lediglich

22 Fischer JURA 2003, 748.

23 Fischer JURA 2003, 748 f; VBlBW 2005, 179; zu weiteren, weniger bedeutenden Fällen, in denen eine Verweisung ausscheidet vgl SCHENKE, Verwaltungsprozessrecht, 11. Aufl 2007, Rdn. 155.

24 Bethge JuS 2001, 1100, 1101; Bethge/von Coelln JuS 2002, 364, 365 Fn. 2.

25 Eine Ausnahme - die im Umkehrschluss die Regel bestätigt - ist explizit in $\$ 50$ III VwGO normiert.

26 Fischer JURA 2003, 748, 749; Kopp/Schenke VwGO, 15. Aufl 2007, $\$ 41 \mathrm{Rdn} .2$ c. AA Sodan/Ziekow/Zie Kow Verwaltungsgerichtsordnung, 2. Aufl 2006, §17 GVG Rdn. 5.

$27 \mathrm{ZB}$ wenn der Kläger ausdrücklich Verpflichtungsklage auf Erlass eines Verwaltungsaktes erhebt, in der Sache aber nur ein Realakt begehrt werden kann. Jedoch darf auch in diesen Fällen nicht die Hinweispflicht des Gerichts nach $\$ 86$ III VwGO übersehen werden; ggf muss der Vorsitzende darauf hinwirken, dass der Antrag geändert wird, vgl Kopp/ScHenKe VwGO, 15. Aufl 2007, \$86 Rdn. 23.

28 Wobei Art. 19 IV GG - ausweislich seines Satzes 2 - nicht erfordert, dass der lückenlose Rechtsschutz gegen die öffentliche Gewalt von der Verwaltungsgerichtsbarkeit gewährt wird; der Gesetzgeber hat sich mit der Formulierung des $\$ 40$ I 1 VwGO aber dafür entschieden, vgl Isensee/ Kirchhof/Papier Handbuch des Staatsrechts Bd. VI, 1. Aufl 1989, \$154 Rdn. 53.

29 Hufen Verwaltungsprozessrecht, 7. Aufl 2008, \$13 Rdn. 2.

$30 \mathrm{Vgl} \mathrm{Sodan/Ziekow/Schmid,} \mathrm{Verwaltungsgerichtsordnung,} \mathrm{2.} \mathrm{Aufl} \mathrm{2006,}$ $\S 88$ Rdn. 5, 18; BVerwG NJW 1977, 1465.

31 FisCHER JURA 2003, 748, 751.

32 Zur historischen Entwicklung des Begriffs der Zulässigkeit FischeR JURA 2003, 748, $749 \mathrm{f}$.

33 Die Zulässigkeit ist vom Gericht stets vorrangig zu prüfen, auch wenn diese schwierige Fragen aufwirft und der Rechtsbehelf offensichtlich unbegründet ist. Dieser Grundsatz beruht darauf, dass die Rechtskraft eines Prozessurteils (Abweisung wegen Unzulässigkeit) weniger weit reicht als die eines Sachurteils. Würde das Gericht die Frage der Zulässigkeit offen lassen, so wäre die Reichweite der Rechtskraft unklar (vgl Kopp/SCHENKe VwGO, 15. Aufl 2007, Vorb $\$ 40$ Rdn. 10).

34 Das gleiche gilt für die Statthaftigkeitsprüfung: Auch insoweit kann das Gericht nur ein Sachurteil fällen, wenn es die richtige Klageart mit ihren spezifischen Zulässigkeitsvoraussetzungen ermittelt hat. 
eine neue Rechtsfolge ${ }^{35}$ für den Fall, dass diese Zulässigkeitsmerkmale fehlen ${ }^{36}$ : Der Rechtsbehelf ist nicht abzuweisen, sondern an das zuständige Gericht zu verweisen. Die allgemeine, ungeschriebene Regel, dass ein Rechtsbehelf abzuweisen ist, wenn er unzulässig ist, gilt nur, solange der Gesetzgeber nicht eine andere Rechtsfolge der Unzulässigkeit eines Rechtsbehelfs normiert ${ }^{37}$.

c) Nach alledem ergibt sich, dass die Aufbauvariante der Vorprüfung nicht empfehlenswert ist. Sie wirft praktische Probleme der Umsetzung auf und führt bei konsequenter Anwendung zu einer noch stärker verunsichernden Umgestaltung des traditionellen Prüfungsschemas. Abgesehen davon basiert sie auf einer nicht überzeugenden Prämisse im Hinblick auf die Definition des Begriffs des Zulässigkeitsmerkmals.

Einer der Zulässigkeit vorgelagerten Vorprüfung bedarf es folglich nicht, da es sich sowohl bei der Rechtswegeröffnung nach $\$ 40$ I 1 VwGO als auch bei der Zuständigkeit weiterhin um Fragen der Zulässigkeit des Rechtsbehelfs handelt.

\section{Sachentscheidungsvoraussetzungen}

Eine weitere Lösung, die von einigen Autoren vertreten wird, ist den Begriff »Zulässigkeit« durch »Sachentscheidungsvoraussetzungen « (oder "Sachurteilsvoraussetzungen «) zu ersetzen ${ }^{38}$. So heißt es dann im Obersatz: »Die Klage ist erfolgreich, wenn die Sachentscheidungsvoraussetzungen vorliegen und sie begründet ist «, statt: »Die Klage ist erfolgreich, wenn sie zulässig und begründet ist «. Folgt man diesem Konzept, so hat dies den Vorteil, dass man nicht viel an dem bekannten Prüfungsaufbau verändern muss, da im Prinzip der Austausch eines Wortes genügt. Gerade wegen seiner Einfachheit hat dieser Vorschlag deshalb seinen Charme, jedoch ist fraglich, ob er wirklich einen Gewinn darstellt.

a) Gegen diesen Aufbau sprechen letztlich dieselben Gründe, die gegen den dreistufigen Aufbau angeführt wurden. Nach diesem Ansatz wird zwischen Sachentscheidungs- und Zulässigkeitsvoraussetzungen differenziert. Der Begriff der Sachentscheidungsvoraussetzungen wird dabei weiter verstanden als der der Zulässigkeit. Zulässigkeitsmerkmale seien nur die Sachentscheidungsvoraussetzungen, deren Nichtvorliegen zur Abweisung des Rechtsbehelfs als unzulässig führe. Demzufolge könnten die Rechtswegeröffnung und Zuständigkeit des Gerichts sich nicht mehr unter den Punkt Zulässigkeit fassen lassen, weil das Fehlen dieser Vorgaben nur die Verweisung an das zuständige Gericht gem. $\$ 17$ a II GVG (i. V. m. $\$ 83$ VwGO) nach sich ziehe. Dies ändere jedoch nichts daran, dass das konkrete Gericht der Verwaltungsgerichtsbarkeit, bei dem der Rechtsstreit anhängig ist, weiterhin die $\$ \$ 40$ I 1,45 und $52 \mathrm{VwGO}$ von Amts wegen zu prüfen habe und nicht in der Sache entscheiden könne, wenn deren Voraussetzungen nicht vorlägen ${ }^{39}$. Letztlich wird als Differenzierungsgrund zwischen den beiden Kategorien also wieder die oben dargestellte (zirkelschlüssige) Definition von Zulässigkeitsmerkmal angeführt ${ }^{40}$. Es überzeugt nicht, alleine mit dieser Argumentation den bislang synonym gebrauchten Begriffen Sachentscheidungs- und Zulässigkeitsvoraussetzungen jeweils eine unterschiedliche Bedeutung beizumessen ${ }^{41}$. Sachentscheidungs- und Zulässigkeitsvoraussetzungen sind und bleiben diejenigen Merkmale, die das Gericht zu prüfen hat, bevor es eine Entscheidung in der Sache treffen kann, unabhängig davon, welche Rechtsfolge ihr Nichtvorliegen hat.

b) Gleichzeitig löst auch dieser Ansatz nicht das Problem des Obersatzes, auf das gleich noch einmal im Zusammenhang mit dem klassischen Aufbau in Zulässigkeit und Begründetheit einzugehen sein wird. Der Rechtsbehelf ist zwar erfolgreich, wenn die Sachentscheidungsvoraussetzungen vorliegen. Er ist andererseits aber nicht unbedingt erfolglos, wenn bestimmte Sachentscheidungsvoraussetzungen fehlen - nämlich die, die die obligatorische Verweisung nach $\$ 17$ a II GVG (i. V.m. $\$ 83$ VwGO) bedingen. Ist dies der Fall, so kann der Rechtsstreit vor dem zuständigen Gericht, zu dem der Rechtsbehelf verwiesen wird, durchaus erfolgreich sein. Daraus folgt, dass der Obersatz so nicht ganz richtig ist.

c) Insgesamt ist folglich auch diese Aufbauvariante abzulehnen. Die Differenzierung zwischen Sachentscheidungs- und $\mathrm{Zu}$ lässigkeitsvoraussetzungen kann nicht überzeugen, vielmehr handelt es sich bei beiden um inhaltlich identische Begriffe.

Selbst wenn man anderer Ansicht ist, kann der Aufbau sein Hauptanliegen nicht erfüllen. Er möchte verdeutlichen, dass der Begriff »Zulässigkeit« zu eng ist, weil er nur diejenigen Sachentscheidungsmerkmale erfasst, die zur Klageabweisung als unzulässig führen. Der Obersatz spiegelt dies aber nicht wider, da er weiterhin impliziert, dass der Rechtsbehelf insgesamt erfolglos ist, wenn die Sachentscheidungsvoraussetzungen nicht vorliegen.

\section{Klassischer Aufbau}

Schließlich ist der klassische Aufbau in Zulässigkeit und Begründetheit zu untersuchen. Wie gezeigt wurde, spricht für diese Aufbauvariante, dass die Änderung von $\$ 17$ a II GVG und $\$ 83$ VwGO nicht dazu geführt hat, dass die Rechtswegeröffnung und die Zuständigkeit nicht mehr als Fragen der Zulässigkeit zu betrachten sind. Die beiden Punkte lassen sich folglich bedenkenlos unter der Überschrift »Zulässigkeit« abhandeln ${ }^{42}$.

35 Zur Ausnahme bei der Rechtswegeröffnung, bei der es bei der Abweisung des Rechtsbehelfs bleibt, weil es sich um eine verfassungsrechtliche Streitigkeit handelt, und daher $\$ 17$ a II GVG keine neue Rechtsfolge bewirkt vgl oben III.1.b)aa).

36 Fischer JURA 2003, 748, 750; Rozek JuS 1996, 87 f; Sodan/Ziekow/SoDAN Verwaltungsgerichtsordnung, 2. Aufl 2006, $\$ 40$ Rdn. 50; Nicht überzeugend ist die Kritik von Hufen Verwaltungsprozessrecht, 7. Aufl 2008, $\$ 10$ Rdn. 1 an dieser Auffassung. Hufen ist der Ansicht, es laufe "auf einen klar erkennbaren Zirkelschluss hinaus, wenn angenommen wird, die Klage sei zwar unzulässig, das Gesetz ordne aber an, dass sie nicht als unzulässig zu behandeln sei«. Damit ist der Inhalt der hier dargelegten Auffassung aber nicht richtig wiedergegeben. Das Gesetz ordnet nicht an, dass eine unzulässige Klage als zulässig zu behandeln sein soll, sondern normiert eine spezielle Rechtsfolge für bestimmte Unzulässigkeitsgründe. Es geht gerade darum, dass die Definition des Begriffs der Zulässigkeit nicht in Abhängigkeit von der Rechtsfolge des Fehlens eines Zulässigkeitsmerkmals erfolgen kann. Hierin lässt sich jedoch kein Zirkelschluss erkennen.

37 FisCHER JURA 2003, 748, 750.

38 Vgl Hufen Verwaltungsprozessrecht, 7. Aufl 2008, $\$ 10$ Rdn. 1; 3. Schmitt Glaeser/Horn Verwaltungsprozessrecht, 15. Aufl 2000, Rdn. 30; Wolff/Decker/Wolf F VwGO/VwVfG Studienkommentar, 2. Aufl 2007, VwGO Vor $\$ 40$ Rdn. 2 (der allerdings den Obersatz widersprüchlich formuliert, vgl unten Fn. 41). Ähnlich wohl auch GEIGER VBlBW 2004, $336 \mathrm{f}$, der von der Prüfung der »Zulässigkeit eines Sachurteils« spricht (kritisch zu dieser weiteren terminologischen Verkomplizierung zu Recht Fischer VBlBW 2005, 179, 180).

39 Hufen Verwaltungsprozessrecht, 7. Aufl 2008, §10 Rdn. 3.

40 So ist wohl Hufen Verwaltungsprozessrecht, 7. Aufl 2008, \$10 Rdn. $3 \mathrm{zu}$ verstehen; dies ist auch die Schlussfolgerung von Sodan/Ziekow/SodAN Verwaltungsgerichtsordnung, 2. Aufl 2006, \$40 Rdn. 50.

41 Für den synonymen Gebrauch vgl Kopp/Schenke, VwGO, 15. Aufl 2007, Vorb $\$ 40$ Rdn. 2. Dass auch Vertreter dieser Aufbauvariante letztlich wieder von der Deckungsgleichheit der Kategorien Sachentscheidungsvoraussetzungen und Zulässigkeit ausgehen, zeigt sich daran, dass sie im Obersatz den Erfolg der Klage von der Zulässigkeit und Begründetheit abhängig machen, dann aber die Prüfung mit "Sachentscheidungsvoraussetzungen « überschreiben (so Wolff/Decker/WoLfF VwGO/ VwVfG Studienkommentar, 2. Aufl 2007, VwGO Vor $\$ 40$ Rdn. 4 f; Lösungsskizze zu Aufgabe 6 aus dem Termin 2000/1 des Bayerischen Ersten Staatsexamens, BayVBl 2002, 57, 61). Es versteht sich von selbst, dass diese inkonsequente Vorgehensweise nicht überzeugen kann und die Verunsicherung im Hinblick auf den richtigen Aufbau weiter verstärkt.

42 Eine andere Frage ist, in welcher Reihenfolge die einzelnen Merkmale innerhalb der Zulässigkeitsprüfung zu erörtern sind, etwa ob Rechtswegeröffnung und Zuständigkeit immer als erstes zu prüfen sind, weil sie nur die obligatorische Verweisung nach $\$ 17$ a II GVG (i. V. m. \$83 VwGO) nach sich ziehen. In der Praxis sollte das Gericht die beiden Punkte schon 
Problematisch ist - wie bereits angesprochen - alleine die Formulierung des Obersatzes. Die Unzulässigkeit eines Rechtsbehelfs hat nicht zwingend zur Folge, dass der Rechtsbehelf abgewiesen wird und damit erfolglos ist. Der Obersatz (für die Klage): »Die Klage ist erfolgreich, wenn sie zulässig und begründet ist « könnte zwar als nicht falsch - denn die Klage ist erfolgreich, wenn sie zulässig und begründet ist - aber als unvollständig angesehen werden, weil er nicht alle Rechtsfolgen einer unzulässigen Klage erfasst. Wollte man ganz exakt arbeiten, so müsste man den Obersatz folgendermaßen formulieren: »Die Klage ist vor dem angerufenen Gericht der Verwaltungsgerichtsbarkeit erfolgreich, wenn sie zulässig und begründet ist«. So ist klargestellt, dass das Gericht bei Unzulässigkeit wegen fehlender Rechtswegeröffnung nach $\$ 40$ I 1 VwGO und fehlender sachlicher oder örtlicher Zuständigkeit die Klage zwar gem. $\$ 17$ a II GVG (i. V.m. $\$ 83 \mathrm{VwGO}$ ) verweisen muss, die Klage aber vor dem konkreten angerufenen Gericht der Verwaltungsgerichtsbarkeit mangels Zulässigkeit keinen Erfolg haben kann. Andererseits ist es aber auch legitim, auf diesen Zusatz zu verzichten und es beim traditionellen Obersatz zu belassen, denn es ist eigentlich selbstverständlich, dass sich die Erfolgsaussichten der Klage immer nur nach der Perspektive des konkret entscheidenden, angerufenen Gerichts bestimmen ${ }^{43}$.

\section{Ergebnis}

Es hat sich gezeigt, dass es insgesamt am überzeugendsten ist, den klassischen Aufbau in Zulässigkeit und Begründetheit beizubehalten. Dies entspricht - dies sei noch erwähnt - auch der ganz herrschenden Ansicht in der verwaltungsprozessualen Literatur ${ }^{44}$, schon deshalb sollten sich die Studierenden durch andere Aufbauvarianten nicht verunsichern lassen. Nichtsdestotrotz sei an die Korrektorinnen und Korrektoren appelliert, gerade bei Aufbaufragen im Interesse der Studierenden keine apodiktische Sichtweise einzunehmen und nur eine bestimmte Variante als »richtig « anzusehen, denn die inhaltlichen Fragen bleiben jeweils dieselben ${ }^{45}$. Sinn und Zweck eines Prüfungsaufbaus ist letztlich, dem Gutachten eine sinnvolle und stringente
Struktur zu geben und eine solche wird von jeder der hier präsentierten Varianten geliefert.

wegen des Rechts auf den gesetzlichen Richter nach Art.101 I 2 GG möglichst zuerst prüfen (allerdings nur soweit dies praktikabel ist, vgl oben unter III.1.a, die Statthaftigkeitsprüfung sollte stets der Zuständigkeit vorgehen), vgl BVerwG NJW 2001, 1513; EhLERs JURA 2007, 830, 833; andererseits aber BVerwG NVwZ 2002, 992, 993; Fischer JURA 2003, 748, 749. Für das Gutachten spielt die Reihenfolge der Zulässigkeitsmerkmale dagegen keine besondere Rolle, da die Erfolgsaussichten der Klage umfassend gutachterlich zu prüfen sind. Insoweit sollte der Aufbau vor allem unter dem Gesichtspunkt der logischen Stringenz und Praktikabilität erfolgen.

43 Anders verhält es sich beim Obersatz der Aufbauvariante "Sachentscheidungsvoraussetzungen«. Hier muss man im Obersatz verdeutlichen, dass es um die Erfolgsaussichten der Klage vor dem angerufenen Gericht der Verwaltungsgerichtsbarkeit geht, denn mit der Differenzierung zwischen Sachentscheidungs- und Zulässigkeitsvoraussetzungen sollen die unterschiedlichen Rechtsfolgen dieser Kategorien gerade verdeutlicht werden.

$44 \mathrm{Vgl}$ nur Schoch/Schmidt-Aßmann/Pietzner/EhLERS Verwaltungsgerichtsordnung, $\$ 41 \S 17$ a GVG Rdn. 5 (die Forderung nach der vorrangige Prüfung der Rechtswegfrage [ a.a. O., Rdn.6] bezieht sich nur auf die Reihenfolge innerhalb der Zulässigkeitsprüfung); DERS JURA 2007, 830, 831; EYERMANN Verwaltungsgerichtsordnung, 12. Aufl 2006, §41 Rdn. 24; Fischer JURA 2003, 748, 750; Kopp/Schenke VwGO, 15. Aufl 2007, $\$ 40$ Rdn. 2 (Zulässigkeits- und Sachentscheidungsvoraussetzungen werden synonym verwendet, vgl a. a. O. Vorb $\$ 40$ Rdn. 2); SCHENKE Verwaltungsprozessrecht, 11. Aufl 2007, Rdn. 65; Sodan/Ziekow/SodAN Verwaltungsgerichtsordnung, 2. Aufl 2006, $\$ 40$ Rdn. 50; WürTENBERGER Verwaltungsprozessrecht, 2. Aufl 2006, Rdn. 120 f; wohl auch STERN/ BLAN Ke Verwaltungsprozessrecht in der Klausur, 9. Aufl 2008 Rdn. 130. $\mathrm{Zu}$ den wenigen Vertretern der aA vgl oben Fn. 38. In Klausurlösungen findet man dagegen die anderen Aufbauvarianten (im Verhältnis zum Meinungsbild in der Kommentar- und Lehrbuchliteratur) leider relativ häufig, was nicht zuletzt zur Verunsicherung der Studierenden beiträgt.

45 In diesem Sinne auch Hufen Verwaltungsprozessrecht, 7. Aufl 2008, §10 Rdn. $1 \mathrm{aE}$, der von einer "friedlichen Koexistenz« der verschiedenen Aufbauvarianten in der Prüfungspraxis spricht. Ähnlich Peine Klausurenkurs im Verwaltungsrecht, 3. Aufl 2008, Rdn.51. Das gilt umso mehr, wenn man die Aufbaufragen für so grundlegend und selbstverständlich hält, dass man deren Erläuterung in der Klausur als Fehler einstuft. Dann muss man alle vertretenen Aufbauvarianten als akzeptable Lösungen anerkennen. 